$(\beta=1.06 ; \quad 95 \% \quad \mathrm{CI}: \quad 0.29, \quad 1.83)$, previous victimization $(\beta=0.33 ; 95 \% \mathrm{CI}: 0.22,0.44)$, and location $(\beta=1.08 ; 95 \% \mathrm{CI}$ : $0.21,1.95)$ were significantly associated with aggressive behavior at Time 2.

Conclusions Previous engagement in aggressive behavior, victimization, and age are all known predictors of aggressive behavior in youth. Youth who report higher levels of rejection sensitivity are more likely to report engagement in aggressive behaviors in the last 3 months.

Significance and Contributions to Injury and Violence Prevention Science Similar to other cognitive behavioral approaches to understanding and reducing youth violence, changing how youth frame and respond to experiences of rejection could reduce engagement in aggressive behaviors.

\section{STUDENT PERCEPTIONS OF LOCKDOWN DRILLS}

Mary Cunningham. University of Michigan School of Public Health

10.1136/injuryprev-2020-savir.129

Statement of Purpose Lockdown or active shooters have become a regular part of youths' school experience, but little research has been done on how these drills affect schools both in terms of preparedness for incidents and effects on the wellbeing of students. We seek to examine correlates of lockdown drill perceptions among a sample of middle school youth.

Methods/Approach As part of a school safety program evaluation, we asked a sample of 6th and 7th grade students a series of questions about lockdown drills in their school. We calculated descriptive statistics to examine the distribution of responses to each item. We plan to conduct further analyses to explore potential correlates of lockdown drill perceptions including victimization, aggressive behavior, rejection sensitivity, depressive symptoms, and demographics.

Results Nearly $90 \%$ of participants agreed that lockdown drills are important to keep students safe. $45 \%$ believed that their peers took the drills seriously and $88 \%$ believed that teachers took the drills seriously. $37 \%$ of students reported that lockdown drills scared them. $80 \%$ of students felt confident that they knew where to go to stay safe in an active shooter emergency. $71 \%$ of students believed that lockdown drills made their school more safe.

Conclusions Overall students had positive perceptions of lockdown drills and their preparedness for an active shooter incident. It is noteworthy that over a third of the sample felt scared by lockdown drills.

Significance and Contributions to Injury and Violence Prevention Science Lockdown drills are conducted to mitigate potential harm in an active shooter event but their effects on reducing or preventing incidents and on student experiences have not been well established. Lockdown drill procedures and components can vary by school district and more research is needed on the implications of different components.

\section{THE HISTORY OF MASS SHOOTINGS}

Ariana Gobaud. 'Columbia University Mailman School of Public Health; ' $G$ Gun Violence Research Center, Rutgers University

10.1136/injuryprev-2020-savir.130
Statement of Purpose Though mass shootings do not account for the greatest number of gun deaths, these incidents garner a great deal of public attention; particularly in light of the disparate media coverage which varies according to victim, location and perpetrator characteristics. The perceived increase in mass shooting incidents may be due to the lack of a standardized definition among media, researchers and law enforcement and consequently, the general population.

Methods/Approach We conducted a narrative review of the literature to understand the evolving definition of mass shooting, the history of the term and specific events that led to paradigm shifts. We coded content for characteristics and patterns of mass shooting events that made them newsworthy.

Results The first documented mass shooting occurred in 1949. The phenomena did not exist before because there was no access to semi-automatic weaponry. The first notable modern mass shooting took place at Columbine High School, an event that remains the most reported mass shooting to date. Over the last 5 years, the use of the term mass shooting has outpaced the true increase in number of events though variation in measurement accounts for the lack of agreement on exactly how much. For example, in 2015, there were either 7, 65, 322, or 371 mass shootings.

Conclusions There is an increased public perception of mass shootings because of a lack of understanding of their real prevalence or their determinants, trends, social costs, and policy implications.

Significance and Contributions to Injury and Violence Prevention Science In order to understand the change in mass shootings over time, a formal definition of the term needs to be established using the same victim, event, location, and perpetrator characteristics to capture the majority of mass shooting events.

\section{APPLICATION OF A RACIAL EQUITY FRAMEWORK FOR GUN VIOLENCE PREVENTION}

Yasmin Fletcher. Educational Fund to Stop Gun Violence

\subsection{6/injuryprev-2020-savir.131}

Statement of Purpose Evidence-based gun violence prevention (GVP) policy reduces firearms-related injury and death by applying research to craft and implement effective policy. This effort to make communities safer must consider and seek to minimize potential unintended outcomes that might negatively impact historically marginalized groups. Making significant impacts in health equity outcomes requires multi-stakeholder engagement. Research forms the foundation of evidence-based interventions and should consider racial equity as it concerns subsequent applications of the work.

Methods/Approach The Educational Fund to Stop Gun Violence (Ed Fund) is a GVP think tank and advocacy organization. We developed a racial equity framework that includes committing ourselves to identifying and addressing inequities across GVP policy and programs as well as evaluating our own organization. We work closely with researchers and encourage a racial equity framework across all areas of work in GVP policy.

Results The Ed Fund: began applying our racial equity framework and process for policy-related work; engaged external 